\title{
Rotation and transposition flaps in facial plastic and reconstructive surgery
}

\author{
Sebastian Sciegienka ${ }^{1}$, Andrea Hanick ${ }^{1}$, Gregory Branham ${ }^{2}$ \\ 'Department of Otolaryngology - Head and Neck Surgery, Washington University in Saint Louis, St. Louis, MO 63110, USA. \\ ${ }^{2}$ Department of Facial Plastic and Reconstructive Surgery, Otolaryngology - Head and Neck Surgery, Washington University in
} Saint Louis, St. Louis, MO 63110, USA.

Correspondence to: Dr. Gregory Branham, Department of Facial Plastic and Reconstructive Surgery, Otolaryngology - Head and Neck Surgery, Washington University in Saint Louis, 660 South Euclid Ave., Campus Box 8115, St. Louis, MO 63110, USA.

E-mail: branhamg@wustl.edu

\begin{abstract}
How to cite this article: Sciegienka S, Hanick A, Branham G. Rotation and transposition flaps in facial plastic and reconstructive surgery. Plast Aesthet Res 2022;9:1. https://dx.doi.org/10.20517/2347-9264.2021.76
\end{abstract}

Received: 25 Jun 2021 First Decision: 28 Sep 2021 Revised: 20 Nov 2021 Accepted: 26 Nov 2021 Published: 7 Jan 2022

Academic Editor: Wen-Guo Cui Copy Editor: Yue-Yue Zhang Production Editor: Yue-Yue Zhang

\begin{abstract}
Reconstructive defects of the human face pose unique challenges to even the most experienced surgeon given their myriad of presentations and the individuality of each patient's anatomy, clinical presentation, and perspective or preferences. A robust armamentarium of reconstructive options must be cultivated for each facial subunit so that experience and artistry can be best utilized to rebuild the patient's structure and function. This review will outline a subset of local rotation and transposition flaps that are most useful for facial reconstruction, organized by facial subunit.
\end{abstract}

Keywords: Rotation, transposition, reconstruction, local flap

\section{INTRODUCTION}

Soft tissue defects of the face, whether post-ablative or traumatic, must be reconstructed with attention to the uniqueness of each defect and each surgical patient. However, understanding the variety of surgical approaches to wound closure can assist the surgeon in choosing the best approach when faced with this challenge. 
Local flaps are often the workhorse of post-Mohs facial reconstruction as they offer many advantages to the patient. Local flaps negate issues associated with a secondary donor site, are often able to replace the skin with like skin, and are capable of being tailored to the specific wound.

Advancement, rotational, and transposition flaps are considered random pattern local flaps, referring to the arterial blood supply of the flap being derived from perforating musculocutaneous blood vessels within the flap's pedicle feeding into the dermal-subcutaneous microcirculatory plexus. This is in contrast to axial pattern local flaps, which derive arterial blood supply from a cutaneous artery that is incorporated into the flap itself. A rotation flap is a random pattern flap that involves the movement of adjacent tissue around an arc of rotation or a pivot point [Figure 1]. The transposition flap is a random pattern flap that involves the movement of tissue across an intervening segment of tissue ${ }^{[1]}$.

In this review, we will focus on options for reconstruction using rotational and transposition flaps. However, it must be acknowledged that in practice, nearly all local flaps involve some degree of both advancement and rotation.

\section{FLAP DESIGN}

When assessing a patient's wound and its closure with local flaps, it is important to appreciate that classic flap designs may need modification to best address each defect. In order to successfully modify the flap to fit the patient's needs, multiple aspects of each wound should be considered. Wound depth and the composition of the missing tissue (skin, subcutaneous fat, muscle, etc.) should be addressed. The tissue's character and quality, including skin laxity, thickness, texture, and the presence of sun damage or postradiation changes, should be noted. The geometry of the flap itself should be optimized to the facial subunit, and the surgeon must choose the orientation of resultant scars that are optimal and ideally in line with relaxed skin tension lines (RSTL).

In addition, the surgeon must consider whether the defect itself should be modified or expanded to create a more ideal reconstructive situation. For example, triangular defects are considered optimal for reconstruction with a classical rotation flap ${ }^{[2]}$. Circular or amorphous defects can be modified into triangular defects to improve the conditions for ideal repair. Complex defects may require a combination approach with multiple local flaps or even the use of a local flap in combination with a skin graft, a pedicled flap, or closure by secondary intention.

Each region of the face has its own risks and benefits to the use of various local flaps due to the inherent characteristics of the skin in the region, specifically skin thickness, texture, elasticity, laxity, and dispensability. For example, the stiffness of the galea and the thickness of the skin in the scalp limit the ability to twist and rotate flaps during scalp reconstruction ${ }^{[3]}$.

\section{ROTATION OR TRANSPOSITION FLAP OPTIONS BY FACIAL SUBUNIT Forehead}

When reconstructing forehead defects, it is key to avoid distortion of the brow position relative to the contralateral side. Anatomically, male patients tend to have thicker brows that rest at the level of the orbital rim, while females tend to have thinner brows that rest above the bony orbital rim with a significant arch that peaks at the level of the lateral limbus or lateral third of the brow. These differences are crucial when reconstructing the brow to achieve the best cosmetic result. The hairline is also an area where symmetry and cosmesis should be considered. Particularly in males, incisions dealing with the hairline should be kept high to accommodate for future hair $\operatorname{loss}^{[4]}$. 


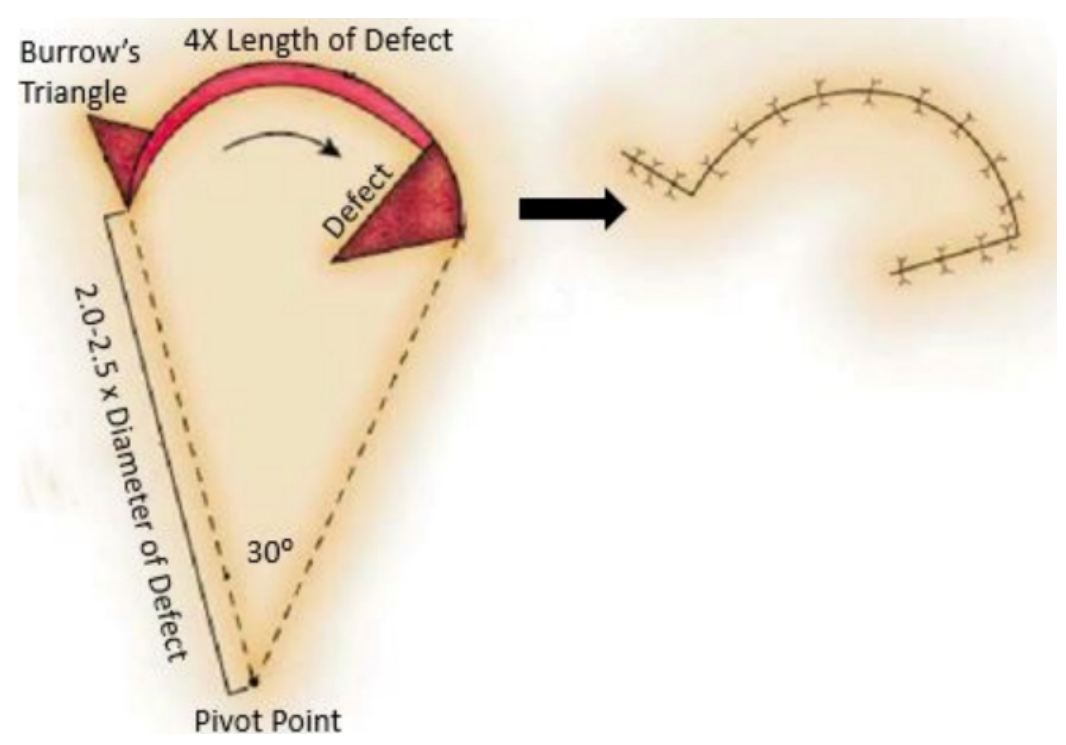

Figure 1. Classic rotation flap. The pivot point is 2.0-2.5 times the diameter of the defect. The distance from the defect's edge to the pivot point is the radius for the rotation flap. This creates an arc of rotation that is approximately 4 times the diameter of the defect. A $30^{\circ}$ rotation is achieved to allow for closure. Arcs of rotation greater than $30^{\circ}$ will generally lead to a standing cone deformity (Burrow's triangle).

The forehead, and its accompanying structures, including the glabella, brow, and temporal region, is comprised of tissue that is less flexible than in other facial subunits making the above goals challenging to achieve. Primary closure is often the best option, but it can only be performed when there is little tension. This is realistic only when there are small defects and extensive subgaleal undermining due to the lack of tissue flexibility. In some patients, closure by secondary intention and/or delayed skin grafting may be the best option but only if there is a vascularized bed of tissue to support the graft without exposed bone. If these options fail, transposition flaps can be a viable reconstructive option. The transposition flap transfers tension of the wound closure from defect to donor site well, but they can easily leave an unacceptable cosmetic result if not planned meticulously. Incisions should be planned in the typically well-defined horizontal RSTL of the forehead. However, this presents a challenge when considering younger patients who have not yet developed forehead rhytids. Secondarily, vertical scars may be cosmetically sound, while diagonal scars are seldom deemed acceptable. In general, the temptation to attempt a local flap on the forehead should only be indulged when all other reconstructive options are eliminated. As such, the following reconstructive techniques are meant to be used as backups to more cosmetically acceptable methods $s^{[4]}$.

\section{$V$ to Y closure}

Although the $\mathrm{V}$ to $\mathrm{Y}$ closure is not a flap per se, it bears mentioning because of its utility in closing glabellar defects. Unlike the forehead, the RSTL of the glabella rests in a vertical orientation. Primary closure of defects could medialize the brow, creating an unnatural appearance. This can be avoided using the $\mathrm{V}$ to $\mathrm{Y}$ technique. In a V to Y closure, a triangular flap is elevated and pulled away from the center of the wound. The lateral aspects of the wound are then brought to the center to create the Y-shaped scar ${ }^{[4]}$.

\section{$O$ to $Y$ and $O$ to $T$}

The $\mathrm{O}$ to $\mathrm{Y}$ and $\mathrm{O}$ to $\mathrm{T}$ are non-linear flap techniques that can be used to close circular defects. The $\mathrm{O}$ to $\mathrm{Y}$ is suitable when the defect is surrounded by relatively elastic skin. The defect is divided into three equal parts, and the underlying tissues are widely undermined to achieve maximal advancement. Maximal tension 
occurs at the center of the wound, where the three parts meet in the center of the defect. The three points should not be directly sutured together as this may strangulate the blood supply to the flap tips. Lastly, one must be cognizant of scar location. This technique will produce a stellate scar and should only be used in situations where this cosmetic result is acceptable. A surgeon would be hard-pressed to place a stellate scar in the flat forehead, but the method may be more cosmetically acceptable near the hairline or temporal tufts $^{[1]}$.

The $\mathrm{O}$ to $\mathrm{T}$ closure is similar to the $\mathrm{O}$ to $\mathrm{Y}$ but is most suitable for defects in which about half of the surrounding skin is significantly more elastic than the other half [Figure 2]. The elastic skin can then be raised as a single flap to close half the defect, converting the arc of the wound edge to the horizontal bar of the $\mathrm{T}$. The remainder of the wound is closed similarly to the $\mathrm{O}$ to $\mathrm{Y}$; two equal flaps are raised, undermined, and brought to the center of the wound, creating the vertical portion of the T-shaped $\operatorname{scar}^{[1]}$. To minimize wound tension, a flap can be constructed in which its height is twice the defect diameter, its base is two defect diameters, and there are roughly three defect diameters of undermining in each direction. These exact measurements will vary depending on defect size, size of standing cones created, and proximity of surrounding structures. Scar placement when performing an $\mathrm{O}$ to $\mathrm{T}$ is an advantage when compared to the $\mathrm{O}$ to $\mathrm{Y}$ flap in the forehead. The base of the scar can be well hidden in the hairline or the brow, leaving only one vertical scar that is less camouflaged, making this technique more useful ${ }^{[5,6]}$.

\section{Cheek}

The cheek is comprised of tissues that are very mobile, and, like the forehead, is relatively flat and featureless. This makes reconstruction more straightforward when compared with the topographic reconstructive challenges of other areas of the face. However, the large, flat plane of the cheek makes camouflaging scars challenging, and thus hiding scars in cheek reconstruction in the boundary of adjacent face subunits is optimal. Despite the lack of true subunits, it is helpful to divide the cheek into different areas: midcheek, inferior, preauricular, melolabial, and infraorbital ${ }^{[7]}$.

In males, the cheek is largely covered with thick, coarse, bearded skin, making scar camouflage more forgiving. In contrast, females' cheek skin is thin and covered only with vellus hairs, making scars are more visible. When reconstructing the cheek, one must be cognizant of the parotid duct and the facial nerve and address them if involved to avoid permanent functional deficit ${ }^{[7]}$.

Although it may be tempting to close cheek wounds primarily because of the tissue's laxity, one must remember that cheek tissues are in constant motion due to talking, chewing, and facial expression. As the tissues flex and relax, healing scars have a tendency to widen. Therefore, cheek closures should be performed in multiple layers; the addition of small skin bandages may also be used to facilitate appropriate healing ${ }^{[7]}$.

\section{Rhombic flaps}

The unirhombic and birhombic transposition flaps are random-pattern local flaps that can be used on small to medium-sized defects that cannot be closed primarily. It is most useful when the surrounding skin is relatively elastic and can be used in many areas of the face, including the cheek, eyelids, chin, forehead, and temple, but we will review their use in the cheek $^{[8]}$. One of the first rhombic transposition flaps described by Alexander Limberg in $1946^{[9]}$. The term Limberg flap was coined to describe his original design consisting of a rhombus-shaped flap with 60 and 120-degree angles that is transposed to cover a similarly shaped defect [Figure 3]. Round defects are trimmed to match the shape of the rhombus prior to the flap's transposition. Variations of the Limberg flap have been developed over the years, including the Dufourmentel flap and 


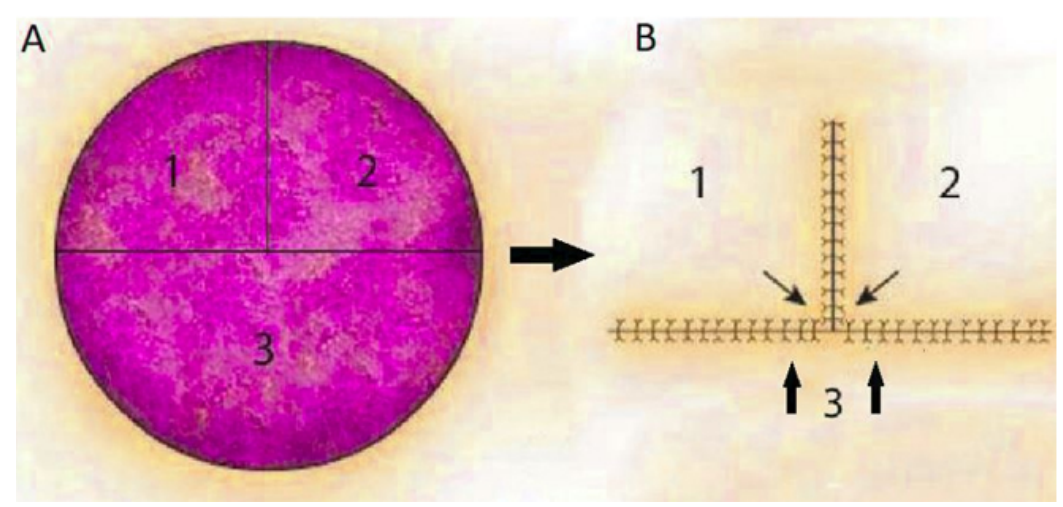

Figure 2. O to T closure. This closure should be designed so that one-half of the defect has greater adjacent skin elasticity (inferior aspect) (A). Half of the closure is accomplished by inferior flap 3 and the other half by superior flaps 1 and 2 equally (B).

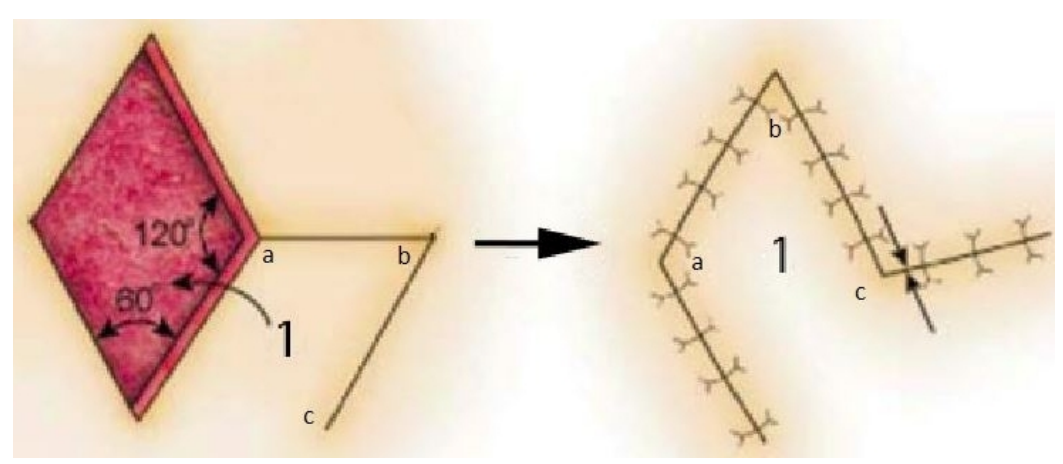

Figure 3. Rhombic flap. The original design by Limberg describes internal angles of $60^{\circ}$ and $120^{\circ}$. Note the transposition of each corner of the rhombic flap labeled a, b, c.

Webster flap, which reduce the tissue needed to close the wound, facilitating wound closure. The Dufourmentel flap is a rhombic flap that consists of any angle combination, while the Webster flap uses a 30-degree angle with a W-plasty at the base of the flap to improve wound closure [Figure 4$]^{[10]}$. Large cutaneous defects can be closed with bilateral rhombic flaps. Using two flaps on opposing sides of the wound allows the surgeon to use elastic or redundant skin from two separate areas to reconstruct the defect $^{[11]}$.

The cosmetic outcome is typically favorable as the transposition of nearby tissues leads to good texture and color match, and the resulting scars have tension vectors that can be predictably placed. When considering scar placement, the short diagonal axis should be oriented perpendicular to the RSTL. Additionally, attention should be paid to facial subunits to avoid crossing or putting tension on neighboring subunits that could distort the area ${ }^{[9]}$. If these principles are followed, the complication rates when using rhombic flaps are similar to other local flaps in the head and neck. However, some have noted a possible slight increase in the rate of trapdoor deformity after healing ${ }^{[12]}$.

\section{Note flap}

The note flap is a triangular transposition flap, given its name based on the appearance of the donor flap, with its defect being similar to a musical eighth note. It allows for a circular defect to be closed with a smaller area of donor tissue. For this reason, it is not recommended for larger defects and is generally felt to be useful in defects less than $2 \mathrm{~cm}^{[13]}$. 


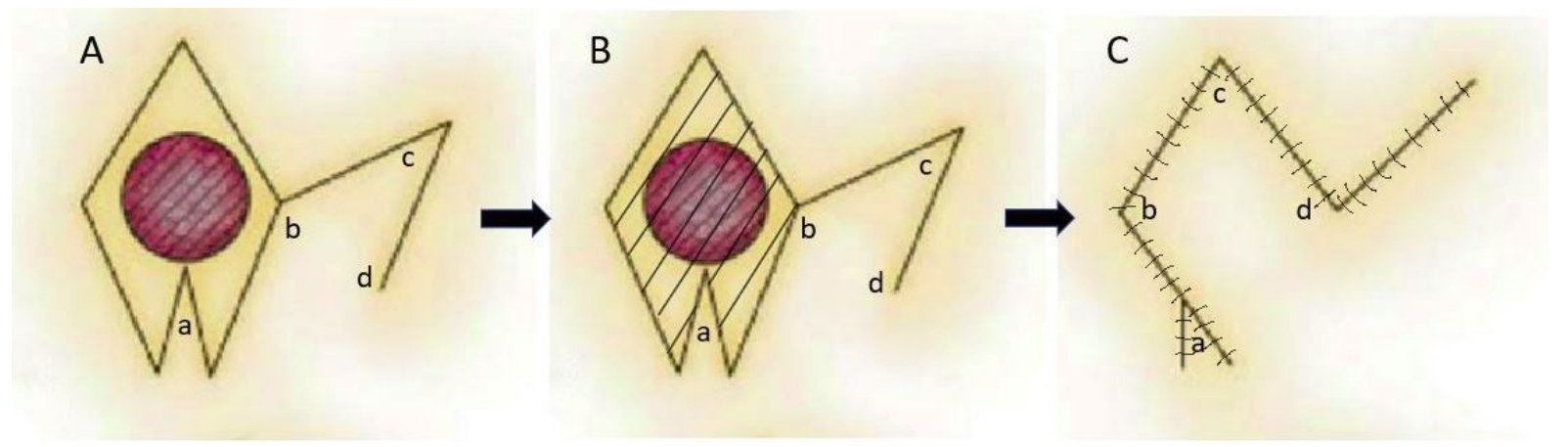

Figure 4. Webster flap. The Webster variation of the classic rhombic flap requires less tissue rotation and reduces wound tension. The flap is designed with a W-plasty inferiorly (A). The hash marks show tissue to be removed prior to the inset of the flap (B). Note the transposition of each corner of the Webster flap labeled a, b, c, d (C).

The flap is designed by first drawing a line tangential to the circular defect along RSTL that is roughly 1.5 times the diameter of the defect. Next, a second line about the length of the defect's diameter is drawn 50 to 60 degrees away from the first to create a triangle. The triangular flap can then be elevated and transposed into a position to close the defect. At times the tip of the triangle may need to be trimmed to facilitate closure $^{[14]}$. Lastly, if a defect is unable to be closed satisfactorily with one note flap, a bilateral note flap can be performed by making the same incisions and transposition opposite to the first.

\section{Cheek rotational flaps}

Larger cheek defects require the transfer of more distant tissue. The cervicofacial flap is useful in such situations because of the excellent color and texture match of the surrounding neck and lateral face skin used in the reconstruction ${ }^{[15]}$. They can be posteriorly or anteriorly based and can be elevated in a subcutaneous or sub-superficial musculoaponeurotic system (SMAS) plane. Some authors advocate for subSMAS dissections in smokers and those who have been irradiated to help prevent flap necrosis. However, others believe that the increased operative time, the risk to the facial nerve, and the technical complexity of sub-SMAS dissection are not outweighed by the theoretical advantage ${ }^{[16]}$.

Cervicofacial flaps are designed in several different ways depending on the degree of tissue advancement and rotation needed to cover the defect. Typically, the incision courses along the superior border of the cheek, inferiorly along the preauricular crease, and around the earlobe toward the hairline. The flap can also be extended inferiorly to the chest in order to recruit more tissue. After broadly raising the flap, it is rotated in position and secured. Large flaps can be quite heavy and can pull surrounding tissue inferiorly if not secured properly. When insetting the flap, it is necessary to tack the flap to the underlying tissues, typically the periosteum, with a bone anchor or a heavy non-absorbable suture. The rest of the closure can proceed in the standard layered fashion ${ }^{[7]}$.

Some anterior cheek or mid-cheek defects can be closed using a bilobed technique. First, the cervicofacial rotation flap recruits all the surrounding cheek tissue to close the primary defect. Then postauricular tissue is used to close the secondary defect along with the preauricular areas.

\section{Eyelid and periocular region}

The complex anatomy, form, and function of the periorbital tissues present a substantial challenge for optimal reconstruction, and numerous techniques have been described to reconstruct the periorbital region and eyelid. In addition to subtle cosmetic implications, improper reconstruction can leave a patient with 
dysfunction or even harmful complications. As such, the following primary goals should be met in any periorbital reconstruction: non-keratinizing epithelium should line the inside of the lid, the eyelid margin must be well defined to avoid hair or lashes touching the eye, the reconstruction should allow for complete eye closure and unobstructed vision with opening, and the eye should look as symmetric to the non-treated eye as possible. The following reconstructive techniques serve as a foundation for periorbital reconstruction to build upon and are not meant to be comprehensive ${ }^{[17]}$.

\section{Tenzel semicircular advancement-rotation flap}

The Tenzel semicircular advancement-rotation flap is useful in repairing central lid defects that are up to $2 / 3$ the length of the lower eyelid and was described in $1975^{[18]}$. An incision is marked from the lateral canthus and extends superiorly and temporally in a curvilinear fashion. The flap is then raised in a submuscular plane with limited use of cautery to preserve blood supply to the flap. Lateral canthotomy and inferior cantholysis are performed. The flap is advanced medially, so that the eyelid margins can be closed on themselves with buried vertical mattress sutures. Prior to closure, the wound edges should be squared to ensure optimal alignment of the eyelid margins, and the closure should be tension-free. After central eyelid reconstruction is complete, the lateral canthal angle must be reconstructed. Typically, the lateral canthal tendon is sutured to the periosteum of Whitnall's tubercle and then to the orbicularis oculi, transposed with the flap to reconstruct the angle. Redundant tissue is removed, and the rest of the lateral flap is tacked down with intradermal sutures secured to the periosteum of the orbit ${ }^{[19,20]}$.

Compared to other methods of eyelid reconstruction, the Tenzel flap is advantageous because it is a onestage operation, often has a less noticeable scar, has minimal donor site morbidity, and effectively prevents lower eyelid ectropion; however, one disadvantage is the lack of lateral eyelashes after the completion of the flap $^{[21]}$.

Fricke flap

The Fricke flap is a forehead and temple-based interpolated transposition flap that originates from above the brow with its base lateral to the lateral canthus. It can be used to reconstruct large laterally positioned upper or lower eyelid anterior lamellar defects. The size of the flap can vary based on the extent of the defect, but in general, the length to width ratio should be 4:1. Furthermore, the flap should be designed to limit the angle of the transposition to less than 90 degrees to avoid vascular compromise. When harvesting the flap, one should avoid dissecting deeply as doing so puts the temporal branch of the facial nerve at risk $^{[22]}$.

The Fricke flap is advantageous in some select scenarios because it is versatile enough to be used in both upper and lower eyelid defects. It also avoids distortion of vision and can support free grafts as it is a vascularized flap. However, its disadvantages make it a somewhat less popular option. The transposition of forehead skin onto the periocular tissues can easily create a mismatch in texture and thickness, and the removal of skin from the forehead will almost certainly create brow height asymmetry. The standard flap also requires a second stage to divide the pedicle several weeks later ${ }^{[2]}$. To circumvent some of these disadvantages, the modified Fricke flap can be used for reconstruction of the lower eyelid. Instead of the forehead skin, the modified Fricke flap uses lateral cheek skin that is typically raised with incisions along RSTL and rotated medially to reconstruct the lower eyelid. The resulting defect can be closed with the relatively relaxed cheek skin using the same principles used with cheek rotation flaps. 


\section{Cheek rotation flap (Mustardé flap)}

The cheek rotational flap, or Mustardé flap, was described by Mustardé in 1971. It is useful when there is a significant vertical defect of the anterior lamella of the lower eyelid that spans $>70 \%$ of its length. It has a wide-based pedicle and excellent vascularity, which makes necrosis of the flap uncommon, and the flap provides an excellent support for free grafts if necessary ${ }^{[23]}$.

An incision is made from the lateral aspect of the defect to the lateral canthus and extends superolaterally, then inferiorly ending at the preauricular area. The flap is elevated in the subcutaneous plane widely to create a tension-free closure. The most medial aspect of the flap should be oriented vertically to reduce the chances of a standing cone. Additionally, a deep inverted triangle may need to be excised inferior to the defect to allow the flap to rotate adequately. The flap is tacked to the medial wall of the orbit medially and the lateral wall of the orbit laterally. Some will place a small vacuum drain in the wound to prevent fluid collection formation ${ }^{[24]}$.

Some disadvantages of the Mustardé technique are the possibility of lower eyelid retraction with scar contracture, ectropion, entropion, and epiphora, as well as risks of facial nerve damage, hematoma formation, and excessive facial scarring.

\section{Lateral orbital flap}

The lateral orbital flap is a pedicled transposition flap that can be used to reconstruct the upper or lower eyelid as well as periocular adnexa, including the eyebrow and orbit in cases of orbital exenteration. As with other eyelid reconstruction techniques, both function and cosmesis is considered when designing the lateral orbital flap. The flap is designed between the lateral canthus and the sideburn as a crescent-shaped island and can be as large as $3 \mathrm{~cm} \times 5 \mathrm{~cm}$. The flap is supplied by the arterial arcade between the zygomatic orbital artery and zygomatic facial artery with or without the inclusion of the named artery, depending on the reconstructive requirements. The pedicle is designed on the medial edge of the flap. Thin flaps containing only skin should be elevated when reconstructing the eyelids, while subcutaneous fat and possibly orbicularis oculi should be elevated with the flap to repair thicker adnexal structures or fill the orbit. Care must be taken when dissecting deep to the SMAS in this location to avoid damaging the frontal branch of the facial nerve. The flap is finally rotated 180 degrees about its medially based pedicle, and the resulting defect is closed primarily resulting in a rather inconspicuous scar. If eyebrow reconstruction is required, flap elevation can extend into the sideburn to capture a small amount of hair-bearing skin; this portion is then transposed into the portion of the missing brow.

Compared to other techniques, the lateral orbital flap is advantageous because it has good color and texture match to the periorbital tissues, can include hair-bearing skin for brow reconstruction, has a rich blood supply making necrosis rare, and the resulting scar is favorable. Disadvantages include the risk to the facial nerve and size limitation of the flap ${ }^{[25,26]}$.

\section{Nose}

Soft tissue reconstruction of the nose presents challenges that are not found in other portions of the face because of its unique topography and deep anatomy. Understanding the nasal subunits is crucial to performing optimal reconstruction and includes the dorsum, paired nasal sidewalls, nasal tip, alar subunits, and columella [Figure 5]. In general, partial subunit reconstruction yields inferior results compared to replacing entire subunits or surgically redefining subunits. One must consider augmenting reconstructive plans if the inner mucosal lining of the nose is affected, which should not be replaced with keratinized $\operatorname{skin}^{[27]}$. 

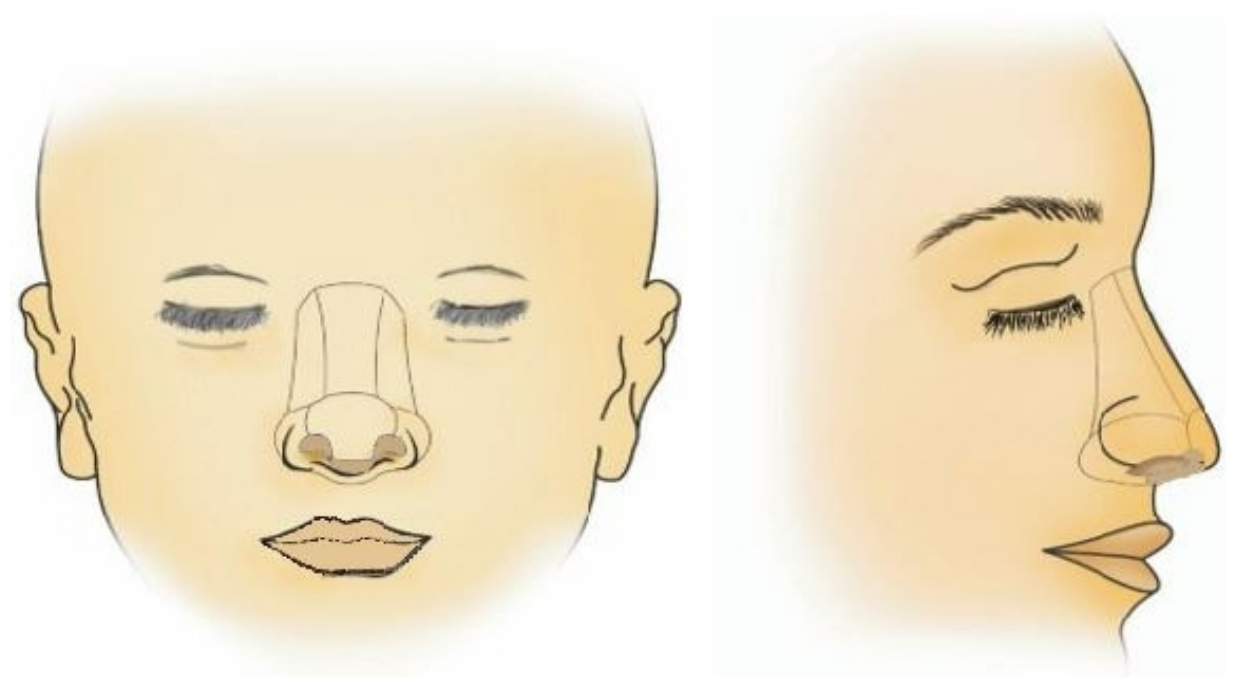

Figure 5. Nasal subunits. The nose can be divided into the tip, dorsal, columellar, and paired sidewall and alar lobules subunits.

The caudal third of the nose is particularly challenging to manage due to its thick, inelastic, and sebaceous skin, unlike the nasal dorsum and sidewalls. The structure of the nasal tip is also relatively fragile and yields to scar contracture forces. Scar contracture secondary to unfavorable healing or poorly planned wound closure can distort the nasal anatomy and lead to poor cosmetic and functional outcomes ${ }^{[28]}$. Healing by secondary intention is acceptable in some areas of the nose, particularly in concave locations such as the medial canthus or upper nasal sidewall. Primary closure may also be utilized when presented with small defects that are surrounded by loose portions of the nasal skin. Ultimately, the full scope of nasal reconstruction exceeds the contents of this chapter. However, we will highlight "workhorse" local flaps that are mainstays of nasal reconstruction.

\section{Sliding glabellar flap (Rieger flap)}

The sliding glabellar flap or Rieger flap is a rotation-advancement flap described in 1967 by Rieger ${ }^{[29]}$ as a method for reconstructing the nasal dorsum, sidewall, lateral nasal tip, and central tip defects in select patients [Figure 6]. One can expect to close defects $2.5 \mathrm{~cm}$ or less using this method. The flap is partially based on an axial blood supply as well as a random blood supply. The base of the flap is contralateral and superolateral to the defect. Prior to performing this flap, soft tissue laxity of the glabella and nasal dorsum should be evaluated to ensure there is enough tissue to provide full defect coverage. Next, a line is created from the lateral extent of the defect up through the nasofacial sulcus, the nasal portion of the medial canthus, and then to a natural glabellar crease. The distance from the base of the glabella to the superior extent of the incision should be 1.5 to 2 times the vertical height of the defect to ensure adequate tissue movement. A back cut to the contralateral medial canthus at about a 45-degree angle should be made. Once the incisions are complete, the tissue is undermined widely in a submuscular (rhinoplasty) plane to allow for maximum rotation. Typically, the flap is secured with a temporary suture to assess for skin thickness discrepancy or distortion of the nasal structure. Underlying tissue can be excised to eliminate height mismatches. The secondary glabellar defect can typically be closed primarily, but V to Y advancement or Zplasty may be necessary. As the flap is rotated downward, care should be taken to remove excess skin and thickness from the tissue that will be adjacent to the medial canthus for maximal cosmesis. The flap is then sutured into place ${ }^{[27,28]}$. 

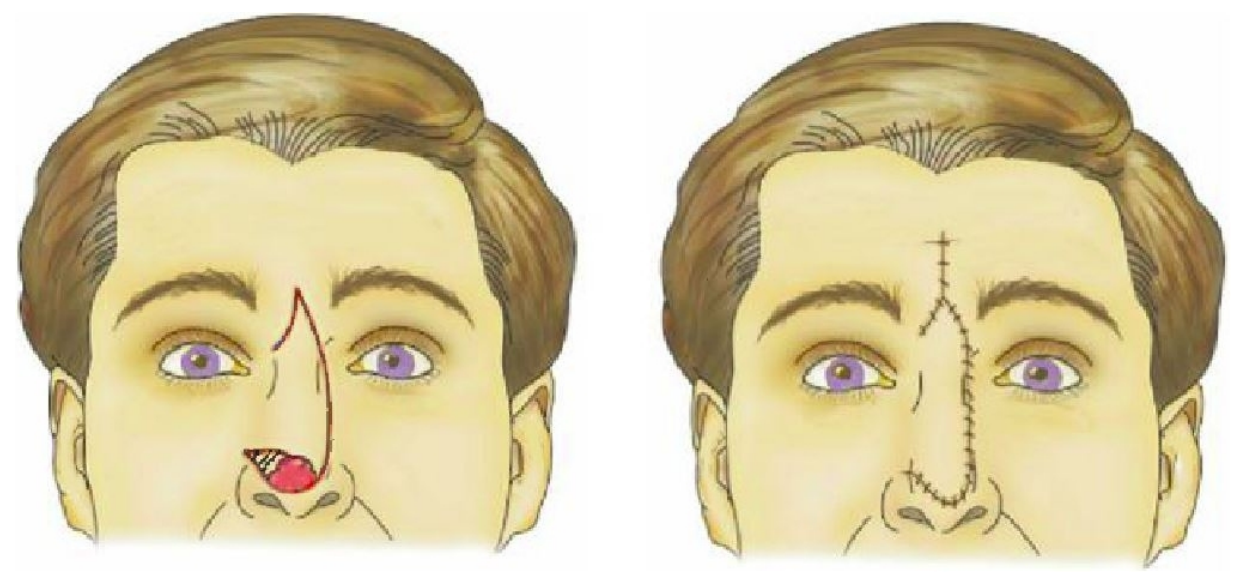

Figure 6. Dorsal nasal flap. The dorsal nasal flap, or Rieger flap, is most useful for lateral defects but can be used for tip defects in select patients.

Minor complications of the Rieger flap include small periorbital hematoma, nasal deformity due to scar contracture, and skin color mismatches. Hematomas, when formed, self-resolve over the following days to weeks. Nasal deformity can be avoided with proper planning and tension-free closure. Lastly, color mismatches or scars can be improved with secondary treatment modalities such as dermabrasion, laser therapy, and steroid injection ${ }^{[28]}$.

The major advantages of this flap are that in a one-stage procedure, like skin is brought into the defect, and incisions can be designed around cosmetic subunit boundary lines.

\section{Bilobed flap}

The bilobed flap is a random pattern, double transposition flap, and is a technique used for small to medium nasal sidewall and nasal tip defects [Figure 7]. Although typically nasal subunits are reconstructed as entire units, some small defects are more appropriately reconstructed with the bilobed flap. It can also be used to reconstruct other areas of the face, such as the $\operatorname{cheek}^{[30]}$. The typical bilobed flap is designed with 90 degrees of total rotation for the primary and secondary flaps. Two flaps are used to redistribute tension over a larger area. The midline of the primary flap is designed about 45 degrees away from the midline of the defect and is usually described as the same size of the primary defect. However, in practice, the primary flap in nasal sidewall reconstruction can be slightly smaller, allowing tissue elasticity to make up the difference in size. When reconstructing the nasal tip, the primary flap and defect sizes should match because of the tight adherence of nasal tip skin to underlying anatomy. When planning local flaps, care should be taken to avoid crossing the cheek-nose border. The secondary flap is oriented 45 degrees from the midline of the defect created by raising the primary flap. It is typically half the size of the primary defect, but in areas where there is more skin elasticity and opportunity to undermine, the size and angle can be adjusted to allow for optimal tissue use and scar placement. Bilobed flaps are advantageous because their excellent color and texture match to nasal defects; however, as with any U- or V-shaped scar, they can be prone to trapdoor deformities ${ }^{[27]}$.

\section{Melolabial flap}

The melolabial flap is a random pattern flap and is a workhorse flap in the reconstruction of the nasal sidewall and lips. It can be based superiorly or inferiorly because of its rich dual blood supply from direct branches of the angular artery and arterial perforators from surrounding muscles. Generally, the angular artery is not incorporated into the flap as a true pedicled flap. Superiorly based flaps are best for large 
A

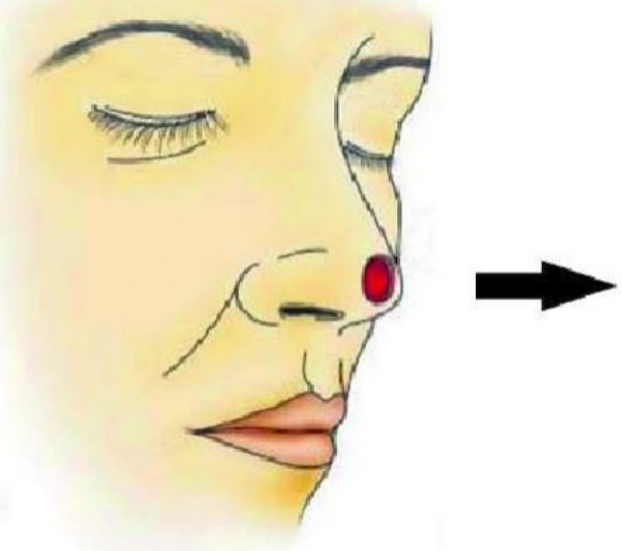

B

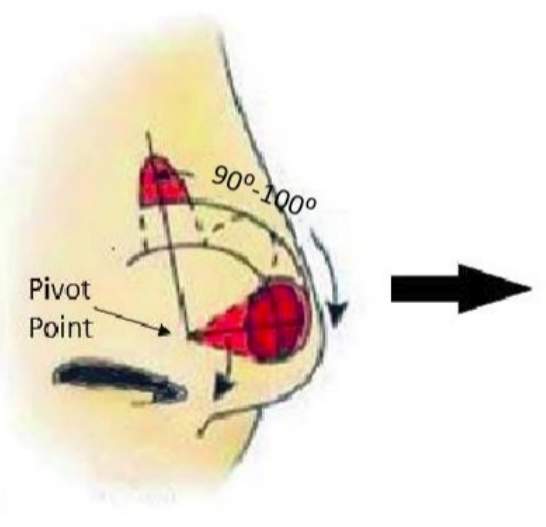

C

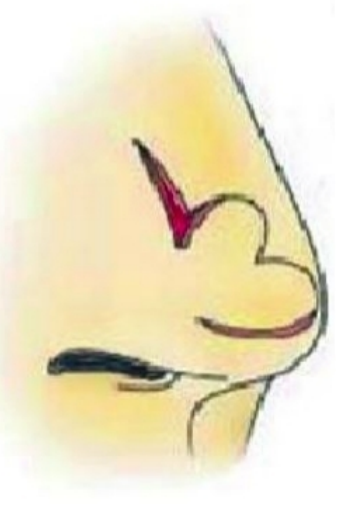

Figure 7. Bilobed flap. Original defect on nasal tip (A). Planned incisions; defect is closed with primary flap, then a secondary flap is used to close the defect created by raising the primary flap (B). Final flap raised (C).
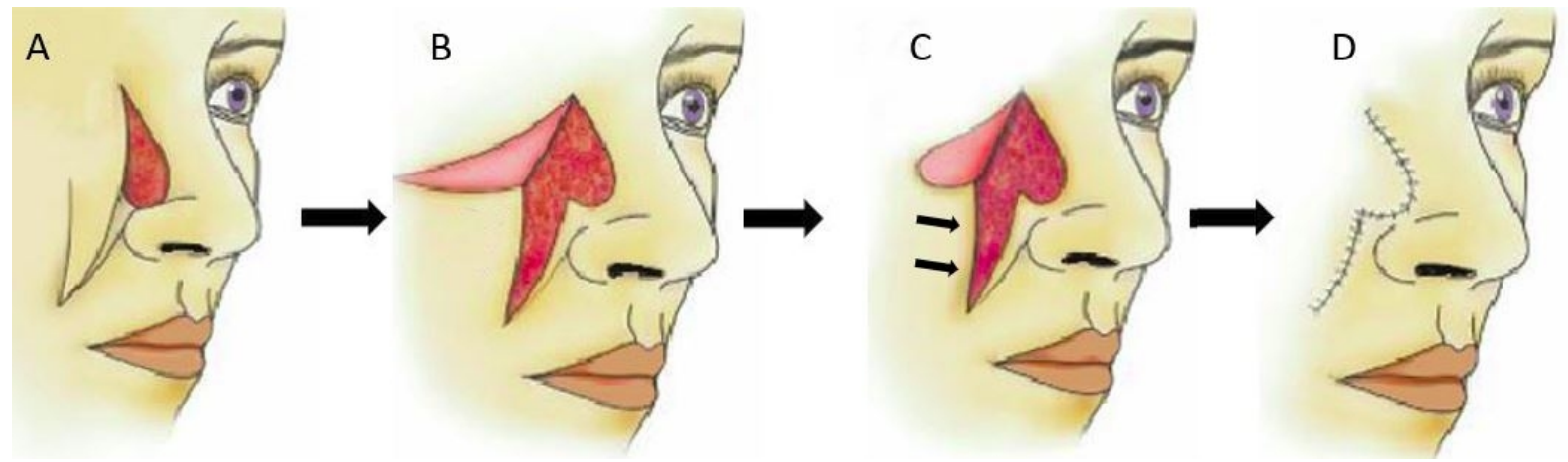

Figure 8. Superiorly based melolabial flap. Original defect on nasal sidewall with planned incisions (A). Flap is raised (B). Flap trimmed to match the size of defect; arrows emphasizing the movement of cheek skin medially to close defect (C). Flap inset with final scar lines shown (D).

A

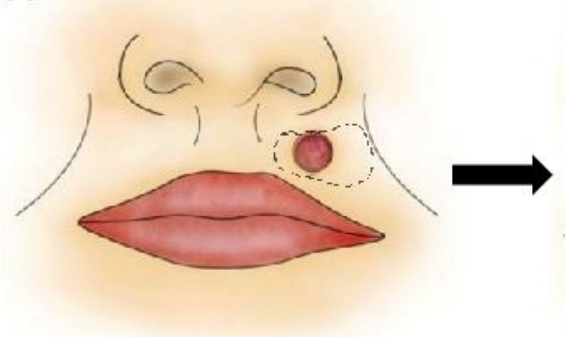

B

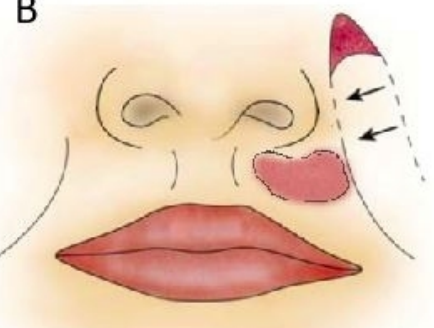

C

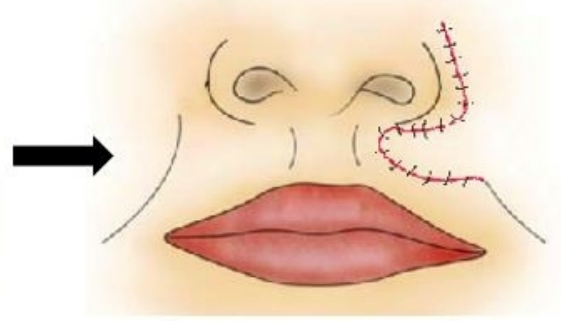

Figure 9. Inferior melolabial flap for lip. Original defect in cutaneous upper lip (A). Defect after resection and planned inferiorly based melolabial flap (B). Final scar lines (C).

defects $(>3 \mathrm{~cm})$, but the dependent position of the distal end of the flap can lead to swelling and lymphedema ${ }^{[27]}$. Superiorly based flaps can also be turned in to reconstruct the nasal lining [Figure 8]. Inferiorly based melolabial flaps are good for small superior defects $(<2 \mathrm{~cm})$, for defects involving the ala, or for upper and lower lip defects [Figure 9] ${ }^{[31]}$. However, an inferiorly based flap can obliterate the alar-facial 


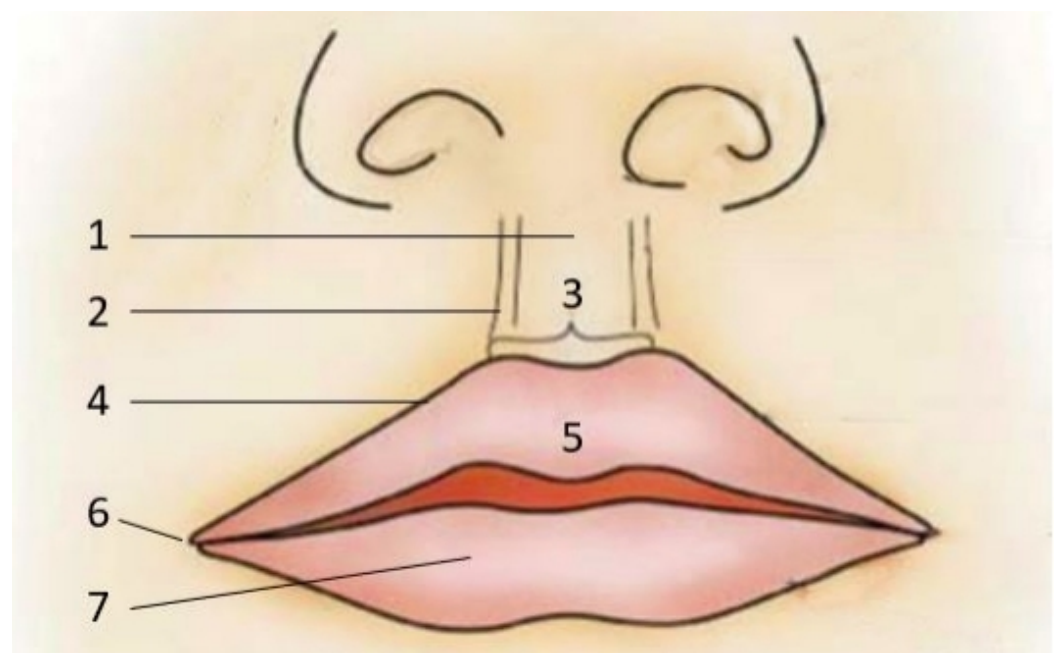

Figure 10. Topographic anatomy of the lips. (1) Philtral groove; (2) Philtral columns; (3) Cupid's bow; (4) White roll of upper lip; (5) Tubercle; (6) Oral commissure; and (7) Vermillion.

groove and are often designed as interpolated flaps, necessitating a second procedure to divide the flap and/or surgically restore the groove.

The melolabial flap should be designed around the melolabial fold and with the recruitment of tissue only from the cheek and never the lip or nose; recruiting tissue from the lip or nose would distort the symmetry of these structures, whereas the laxity of the cheek allows for much greater tissue utilization without cosmetic consequence ${ }^{[27]}$. When designing the flap, the melolabial flap is first marked. Then a template is made of the defect and is used to mark out the amount of tissue necessary for reconstruction. A $4 \times 4$ gauze is useful to measure the length of the flaps designed relative to the location of the defect, pivoting around the base of the flap. The pedicle width should be between 1.5 and $2 \mathrm{~cm}$ to maintain perfusion. Incisions are then made, and the flap is elevated off the cheek. The flap is elevated with gradually increasing thickness such that the distal end of the flap is thinnest and the base is the thickest. The flap is then secured into position. Donor site closure is typically uncomplicated due to cheek laxity, but one should ensure that surrounding structures such as the eyelid or lip are not being distorted as a result of tight closure ${ }^{[32]}$.

\section{Lips}

The lips are the central point of the face because of their role in expression and communication. They are both cosmetically sensitive and play a major functional role in speech and eating. The primary objective of lip reconstruction is the restoration of oral competence followed by maintenance of muscular continuity, sensation, and oral aperture circumference. Generally, the lip complex should be used to repair defects to maintain oral competence; if using the lip complex alone would lead to microstomia, adjacent tissues are included in the reconstruction. Lastly, the cosmetic appearance of the lip and the proportions of the upper and lower lip should be considered with all lip reconstructions. The multilaminar anatomy and lack of rigid underlying structures make the above goals challenging to the reconstructive surgeon ${ }^{[33]}$.

Oral and perioral defects can be categorized by their size and location. As with other areas of the face, the lips are comprised of discrete subunits and follow the same subunit principles discussed previously. The subunits of the lips include the philtrum, Cupid's bow, white roll of the upper lip, tubercles, commissures, vermillion, and upper and lower cutaneous lip [Figure 10]. Small lesions on the cutaneous lip far from the white roll can heal via secondary intension; this method should be avoided if the defect is close to the white 
roll due to the possibility of retraction and lip deformity after healing. Full-thickness defects can often be closed primarily if they involve less than one-third of the width of the lower lip. Larger defects often necessitate the use of local flaps. Rearrangement of lip tissue utilizing advancement flap techniques such as the Karapandzic flap or pedicled flap techniques such as the Abbe and Estlander flaps allow for the replacement of like tissue with like tissue. However, in cases where existing lip structure is lacking to the point that more distant tissue must be recruited, options such as the perialar crescentic advancement flap are useful. Mucosal advancement flaps are often used in conjunction with local skin flaps to reconstruct the mucosal components of multilaminar, full-thickness defects. The rotational flap most utile for reconstruction is the inferiorly based melolabial flap which has been described in a previous section.

\section{CONCLUSION}

Facial reconstruction requires careful consideration of the unique qualities of each facial subunit and the corresponding advantages and risks of each type of reconstructive option. Often, local skin flaps provide the best result with the least morbidity. As described, rotation and transposition flaps can be utilized successfully for facial reconstruction when the flap is chosen correctly and is well designed and executed.

\section{DECLARATIONS}

\section{Authors' contributions}

Made substantial contributions to conception and design of the study and performed data review and drafted the manuscript text and created the figures: Sciegienka S

Made substantial contributions to conception and design of the study and performed data review and edits of manuscript: Hanick A

Made substantial contributions to conception and design of the study and provided insights and final edits to the manuscript: Branham G

\section{Availability of data and materials}

Not applicable.

\section{Financial support and sponsorship}

None.

\section{Conflicts of interest}

All authors declared that there are no conflicts of interest.

\section{Ethical approval and consent to participate}

Not applicable.

\section{Consent for publication}

Dr. Gregory Branham (corresponding author) has given permission to use the figures.

\section{Copyright}

(c) The Author(s) 2022.

\section{REFERENCES}

1. Branham GH. Principles of flap design and preoperative analysis. In: Branham G, Thomas JR, editors. Facial soft tissue reconstruction: Thomas procedures in facial plastic surgery. Shelton, CT: People's Medical Pub. House; 2011. p. 31-51.

2. Baker SR. Rotation flaps. Local flaps in facial reconstruction. 3rd ed. Philadelphia, PA: Elsevier/Saunders; 2014 . p. 100-23.

3. Earnest LM, Byrne PJ. Scalp reconstruction. Facial Plast Surg Clin North Am 2005;13:345-53, vii. DOI PubMed

4. Branham GH. Forehead and Brow Reconstruction. In: Branham G, Thomas JR, editors. Facial soft tissue reconstruction: Thomas procedures in facial plastic surgery. Shelton, CT: People's Medical Pub. House; 2011. p. 89-96. 
5. Stevens CR, Tan L, Kassir R, Calhoun K. Biomechanics of A-to-T flap design. Laryngoscope 1999;109:113-7. DOI PubMed

6. Krishnan R, Garman M, Nunez-Gussman J, Orengo I. Advancement flaps: a basic theme with many variations. Dermatol Surg 2005;31:986-94. DOI PubMed

7. Branham GH. Cheek reconstruction. In: Branham G, Thomas JR, editors. Facial soft tissue reconstruction: Thomas procedures in facial plastic surgery. Shelton, CT: People's Medical Pub. House; 2011. p. 97-103.

8. Tamborini F, Cherubino M, Scamoni S, Frigo C, Valdatta L. A modified rhomboid flap: the "diamond flap". Dermatol Surg 2012;38:1851-5. DOI PubMed

9. Macneal P, Adlard RE. Rhombic flaps. In: StatPearls [Internet]. Treasure Island, FL: StatPearls Publishing; 2021.

10. Baker SR. Rhombic flaps. In Baker SR, editor. Local flaps in facial reconstruction. 3rd ed. Philadelphia, PA: Elsevier/Saunders; 2014. p. 204-21.

11. Johnson TM, Wang TS, Fader DJ. The birhombic transposition flap for soft tissue reconstruction. J Am Acad Dermatol 1999;41:232-6. DOI PubMed

12. Sclafani AP, Sclafani JA, Sclafani AM. Successes, revisions, and postoperative complications in 446 Mohs defect repairs. Facial Plast Surg 2012;28:358-66. DOI PubMed

13. Baker SR. Transposition flaps. 3rd ed. Philadelphia, PA: Elsevier/Saunders; 2014. p. 131-55.

14. Walike JW, Larrabee WF Jr. The 'note flap'. Arch Otolaryngol 1985;111:430-3. DOI PubMed

15. Heller L, Cole P, Kaufman Y. Cheek reconstruction: current concepts in managing facial soft tissue loss. Semin Plast Surg 2008;22:294-305. DOI PubMed PMC

16. Pepper JP, Baker SR. Local flaps: cheek and lip reconstruction. JAMA Facial Plast Surg 2013;15:374-82. DOI PubMed

17. Baker SR. Reconstruction of the eyelids. In: Baker SR, editor. Local flaps in facial reconstruction. 3rd ed. Philadelphia, PA: Elsevier/Saunders; 2014. p. 371-98.

18. Tenzel RR. Reconstruction of the central one half of an eyelid. Arch Ophthalmol 1975;93:125-6. DOI PubMed

19. Patel SY, Itani K. Review of eyelid reconstruction techniques after Mohs surgery. Semin Plast Surg 2018;32:95-102. DOI PubMed $\mathrm{PMC}$

20. Branham GH. Eyelid and periocular reconstruction. In: Gregory Branham, J. Regan Thomas, editors. Facial soft tissue reconstruction: Thomas procedures in facial plastic surgery. Shelton, CT: People's Medical Pub. House; 2011. p. 105-15.

21. Cha JA, Lee KA. Reconstruction of periorbital defects using a modified Tenzel flap. Arch Craniofac Surg 2020;21:35-40. DOI PubMed PMC

22. Wilcsek G, Leatherbarrow B, Halliwell M, Francis I. The 'RITE' use of the Fricke flap in periorbital reconstruction. Eye (Lond) 2005;19:854-60. DOI PubMed

23. Mustardé JC. Repair and reconstruction in the orbital region: a practical guide. 2d ed. Edinburgh; New York, New York: Churchill Livingstone; distributed in U.S. by Longman; 1980. p. 400.

24. Mustardé JC. Major reconstruction of the eyelids: functional and aesthetic considerations. Clin Plast Surg 1981;8:227-36. PubMed

25. Matsuo S, Hashimoto I, Seike T, Abe Y, Ishida S, Nakanishi H. Extended hair-bearing lateral orbital flap for simultaneous reconstruction of eyebrow and eyelid. Plast Reconstr Surg Glob Open 2014;2:e111. DOI PubMed PMC

26. Ogawa Y, Takemoto T, Suzuki K, Kusumoto K. Application of the lateral orbital flap to reconstruction of the upper and lower eyelids and the eye socket after enucleation. Ann Plast Surg 2011;66:360-3. DOI PubMed

27. Branham GH. Nasal reconstruction. In: Branham G, Thomas JR, editors. Facial soft tissue reconstruction: Thomas procedures in facial plastic surgery. Shelton, CT: People's Medical Pub. House; 2011. p. 59-78.

28. Johnson TM, Swanson NA, Baker SR, Brown MD, Nelson BR. The Rieger flap for nasal reconstruction. Arch Otolaryngol Head Neck Surg 1995;121:634-7. DOI PubMed

29. Rieger RA. A local flap for repair of the nasal tip. Plast Reconstr Surg 1967;40:147-9. DOI PubMed

30. Dvořák Z, Vavrek V, Kubek T, Streit L, Stupka I, Veselý J. Bilobed flap in facial reconstruction. Acta Chir Plast 2020;61:10-5. PubMed

31. Baker SR. Melolabial flaps. In Baker SR, editor. Local flaps in facial reconstruction. 3rd ed. Philadelphia, PA: Elsevier/Saunders; 2014. p. 231-67.

32. Jewett BS. Interpolated forehead and melolabial flaps. Facial Plast Surg Clin North Am 2009;17:361-77. DOI PubMed

33. Branham GH. Lip and perioral reconstruction. In: Branham G, Thomas JR, editors. Facial soft tissue reconstruction: Thomas procedures in facial plastic surgery. Shelton, CT: People's Medical Pub. House; 2011. p. 79-87. 